\title{
Differential Scanning Calorimetry and Stress Relaxation of Partially Saponificated Poly(vinyl alcohol) -Dimethyl Sulfoxide-Water System
}

\author{
Mineo WATASE and Katsuyoshi NiSHINARI*.† \\ Chemical Research Laboratory, Faculty of Liberal Arts, \\ Shizuoka University, Ohya, Shizuoka 422, Japan \\ * Instrumentation Research Laboratory, National Food Research Institute, \\ Ministry of Agriculture, Forestry and Fisheries, \\ Tsukuba 305, Japan
}

(Received December 1, 1988)

\begin{abstract}
In order to clarify the relation between the structure and properties of poly(vinyl alcohol) (PVA) gel, differential scanning calorimetry (DSC) and stress relaxation measurements were carried out. A small and broad endothermic peak which appeared at about $45^{\circ} \mathrm{C}$ was attributed to the disentanglement of molecular chains. A sharp endothermic peak which appeared in the temperature range from $70^{\circ} \mathrm{C}$ to $110^{\circ} \mathrm{C}$ was attributed to the gel melting or transition from gel to sol. The endothermic enthalpy determined from the area enclosed by the melting endothermic peak and the beseline showed a maximum at a DMSO concentration around $0.277 \mathrm{mf}$, which was almost independent of the concentration of PVA. The relaxation spectrum of PVA gels showed a maximum as a function of DMSO concentration at $0.277 \mathrm{mf}$ DMSO. It was suggested that the number and structures of junction zones and the conformations of flexible chains connecting junction zones are optimized to give maxima of the elastic modulus and the endothermic enthalpy of melting.

KEY WORDS Gel / Partially Saponificated Poly(vinyl alcohol) / Dimethyl

Sulfoxide / Stress Relaxation / DSC / Water /
\end{abstract}

Physico-chemical properties of poly(vinyl alcohol) (PVA) gels prepared by repeating freezing-thawing cycles have been studied because these gels can be used in bio-medical industries and are also interesting model substances in order to clarify the gelation mechanism and structures of hydrophilic polymers. ${ }^{1-8}$ When the degree of saponification (DS) is higher than $96 \mathrm{~mol} \%$, and the concentration of PVA is high enough, very strong gels are formed with mechanical properties similar to commercially available rubber stoppers. ${ }^{5}$ PVA with low DS does not form a strong gel below the concentration $30 \%(\mathrm{w} / \mathrm{w})$ even though the solution is subjected to freezing-thawing cycles. ${ }^{5}$ PVA with higher DS ( $>96 \mathrm{~mol}^{\circ} \%$ ) forms a strong gel even at a low concentration as $15 \%(\mathrm{w} / \mathrm{w})$ when its solution is subjected to freezing-thawing cycles. In contrast, when DMSO is added to aqueous solution of PVA, a strong gel is formed even if the DS is low.

PVA gels are believed to consist of crystalline and amorphous regions. A crystalline region consists of junction zones, aggregation of ordered molecules, while an amorphous region consists of long flexible molecules. ${ }^{9.10}$ The molecular forces which make the crystalline region are believed to be mainly hydrogen bonds rather than van der Waals forces. The

\footnotetext{
† To whom correspondence should be addressed.
} 
presence of DMSO changes the structures of junction zones and the conformation of long flexible chains which connects junction zones. As a result, physico-chemical properties of PVA-DMSO-water gels will change. This change has been studied by rheological and thermal measurements in the present work.

\section{EXPERIMENTAL}

\section{Materials}

The degree of polymerization (DP) and DS of PVA used in this study were 300 and $88 \pm 0.5 \mathrm{~mol} \%$, respectively. DMSO of the extra fine grade reagent (Wako Pure Chemical Industries, Ltd.) was used without further purification. PVA solutions were molded to cylindrical gels $(20 \mathrm{~mm}$ diameter and $30 \mathrm{~mm}$ length) as described previously. ${ }^{11}$

\section{Stress Relaxation}

The apparatus used for stress relaxation measurements was a chainomatic balance type relaxometer, which was used in previous studies. ${ }^{11}$ Relaxation curves up to 10 hours were observed in the temperature range from $15^{\circ} \mathrm{C}$ to $75^{\circ} \mathrm{C}$ controlled by a silicone oil bath.
Strain was always $5 \%$ throughout this study.

\section{Differential Scanning Calorimetry}

Differential scanning calorimetry (DSC) of gels was carried out by a Sensitive DSC SSC $560 \mathrm{U}$ (Seiko Instruments \& Electronics, Ltd.). Forty five mg each of the gels were sealed into silver pans of $70 \mu \mathrm{l}$. A DMSO solution was used as a reference, and the weight was made equal within $\pm 0.1 \mathrm{mg}$ to that of the sample gel in order to obtain a flat base line. The temperature was raised from $2 \mathrm{C}$ at a heating rate of $2 \mathrm{C} \mathrm{min}^{-1}$ to observe the endothermic peak accompanying the transition from gel to sol.

\section{RESULTS AND DISCUSSION}

Figures 1(A)-(C) show typical heating DSC curves of PVA gels of various concentrations for different DMSO concentrations in DMSO-water. PVA gels are be- lieved to consist of somewhat crystalline junction zones and amorphous regions. ${ }^{9.10}$ The junction zone is considered to consist of a parallel aggregation of extended molecular chains, while the amorphous region is considered to consist of entangled flexible chains.

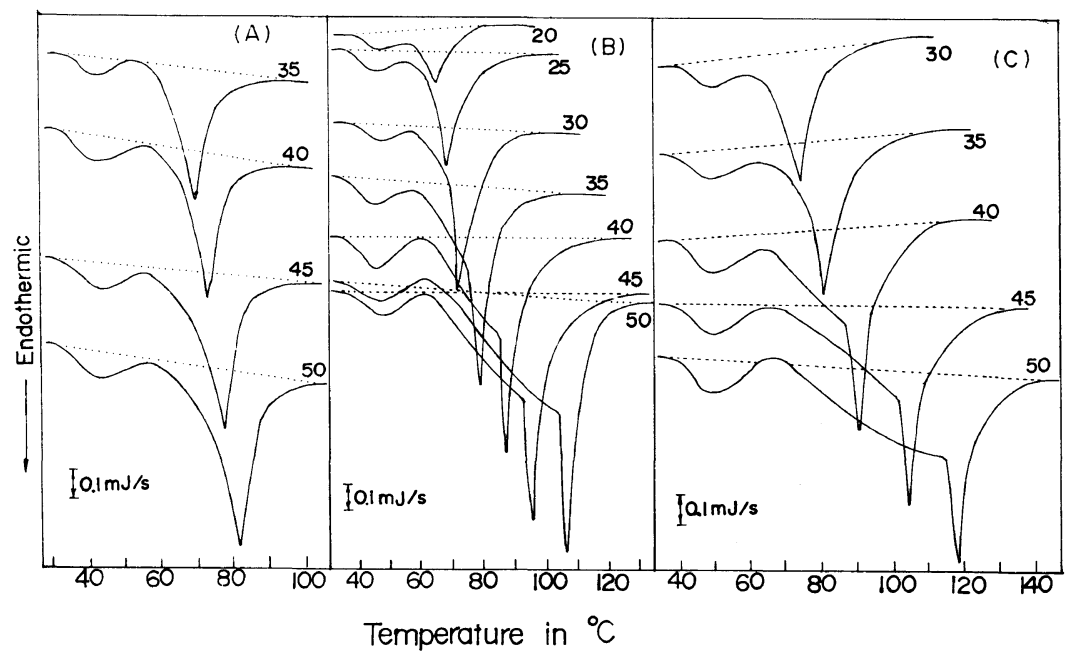

Figure 1. Heating DSC curves of PVA-DMSO-water gels of various concentrations. Figures beside each curve represent the concentration of PVA in $\%(w / w) . D P=300, D S=88 \mathrm{~mol} \%$. DMSO concentration: (A), $0.095 \mathrm{mf}$; (B), $0.277 \mathrm{mf}$; (C), $0.582 \mathrm{mf}$. 
The energy required for molecular motion is supposed far lower for molecular chains in amorphous regions than for molecular chains in crystalline regions. A small and broad endothermic peak which appeared at about $45^{\circ} \mathrm{C}$ was attributed to the disentanglement of molecular chains. This small endothermic peak was found only in PVA gels of partial saponification (DS $=88 \mathrm{~mol} \%$ ) and was not found in PVA gels of complete saponification (DS $>96$ $\mathrm{mol} \%$ ). The reason is considered as follows: PVA gels of complete saponification consist mainly of crystalline regions, while PVA gels of partial saponification consist mainly of amorphous regions. A sharp endothermic peak which appeared at the temperature range from $70^{\circ} \mathrm{C}$ to $110^{\circ} \mathrm{C}$ was attributed to the melting of gel or the transition from gel to sol. This transition temperature is called $T_{\mathrm{m}}$ hereafter. A small and broad endothermic peak appeared at about $45^{\circ} \mathrm{C}$ was not affected so much by the concentration of added DMSO, while $T_{\mathrm{m}}$ shifted to higher temperatures up to a certain DMSO concentration and beyond that DMSO concentration, it shifted to lower temperatures with increasing DMSO.

The melting temperature $T_{\mathrm{m}}$ of PVADMSO-water gels as a function of DMSO concentration takes a maximum around 0.277 mf DMSO for $20-25 \%$ (w/w) PVA gels, and the DMSO concentration giving the maximum $T_{\mathrm{m}}$ increased with increasing concentration of PVA (Fig. 2). This tendency is contrary to that observed in agarose-DMSO-water gels. ${ }^{12}$ From the experimental observation that agarose molecules do not form a strong gel in DMSO, it was suggested that agarose molecules cannot form junction zones in DMSO, while they form junction zones in water. In the present case of partially saponificated PVA, water is not a single solvent to form a strong gel. DMSO is necessary for partially saponificated PVA to form a gel in the present case. Kajiwara et al. ${ }^{13}$ showed that $1-5 \%(\mathrm{w} / \mathrm{w})$ PVA with higher DS $(99.5 \mathrm{~mol} \%$ ) forms a gel in the presence of DMSO concentration range

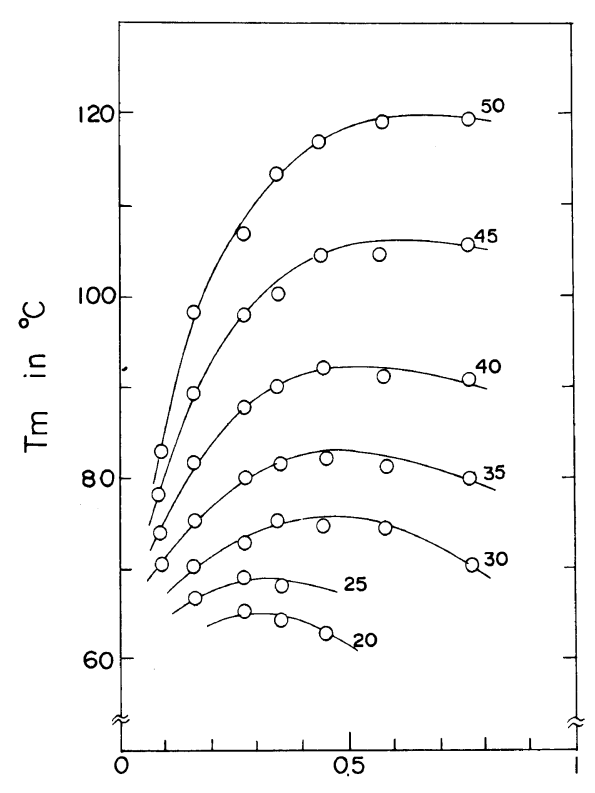

DMSO concentration in mole fraction

Figure 2. Melting point $T_{\mathrm{m}}$ of PVA-DMSO-water gels of various PVA concentrations as a function of DMSO concentration. Figures beside each curve represent the concentration of PVA in $\%(w / w)$.

from 40 to $80 \%$ (w/w). They found beyond this DMSO concentration the PVA-DMSO-water system forms a transparent solution, and that below this DMSO concentration this system is not homogeneous and some cloud-like clusters float. They also observed that this system forms an opaque gel with the DMSO concentration less than $40 \%(\mathrm{w} / \mathrm{w})$, because water is a poor solvent for PVA, and thus it prevents PVA chains to swell. Therefore, the experimental fact that the maximum $T_{\mathrm{m}}$ appeared in higher DMSO concentrations with increasing concentration of PVA may be understood from the difference of dissolving power of DMSO and water for polymers.

Figure 3 shows the endothermic enthalpy $\Delta H_{\mathrm{m}}$ which was determined from the area enclosed by the sharp endothermic peak and the base line (dotted line in Figures 1(a)-(c)) as a function of DMSO concentrations for PVA gels of various concentrations. $\Delta H_{\mathrm{m}}$ showed a 
maximum at about $0.277 \mathrm{mf}$ DMSO irrespective of PVA concentrations.

Reduced relaxation Young's modulus of $40 \%(\mathrm{w} / \mathrm{w})$ PVA gels at various temperatures is shown for different DMSO concentrations in Figure 4.

Master relaxation curves were made from reduced relaxation Young's modulus by means of a reduced variable method, and are shown in Figure 5. Relaxation spectra were determined using Alfrey's approximation, and are shown in Figure 6. Both in Figures 5 and 6,

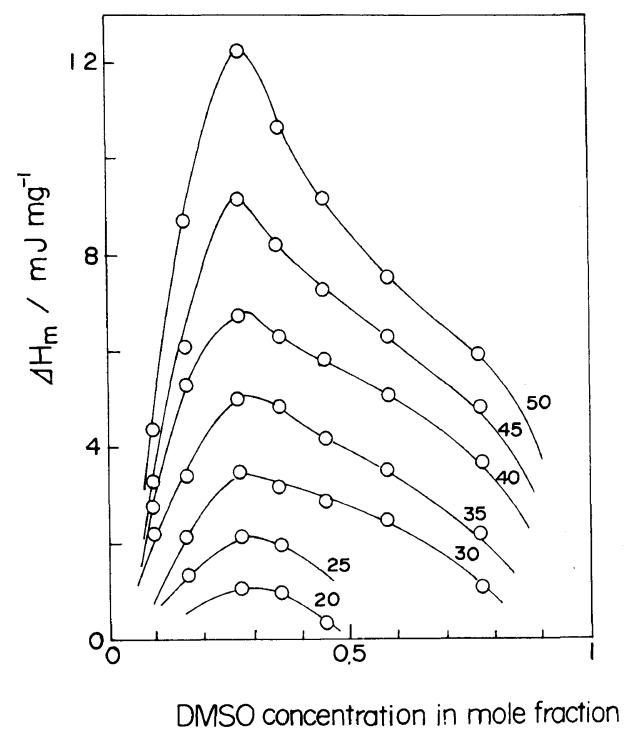

Figure 3. The relation between endothermic enthalpy $\Delta H_{\mathrm{m}}$, determined from the area enclosed by the melting endothermic peak and the baseline, and DMSO concentration. master relaxation curve or relaxation spectrum as a function of DMSO concentration is largest at DMSO concentration of $0.277 \mathrm{mf}$. The shift factors are plotted against the inverse of the absolute temperature in Figure 7 . The apparent activation energy was estimated as $191.5 \mathrm{~kJ} \mathrm{~mol}^{-1}$ from the slope of the straight line part of this plot. This value was almost independent of DMSO concentration.

Generally speaking, the state of gels depends

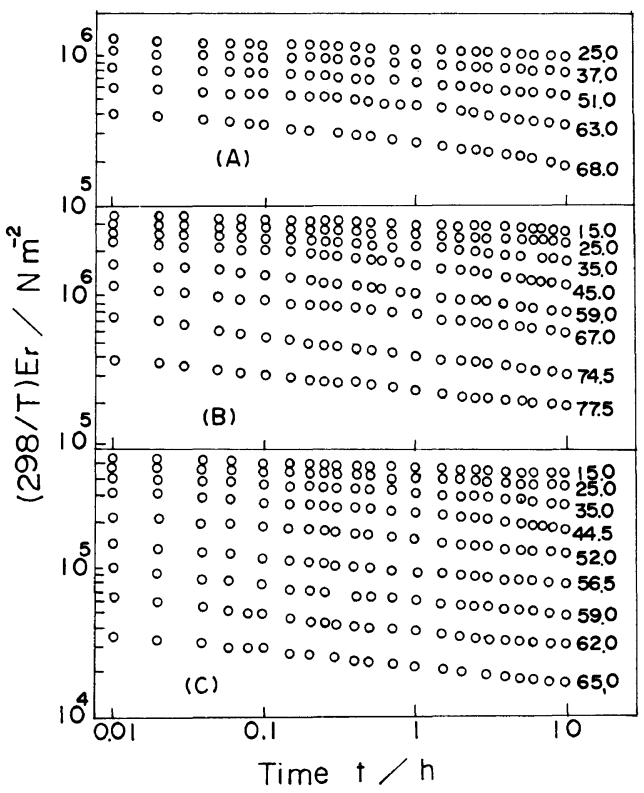

Figure 4. Reduced relaxation Young's modulus of $40 \%$ (w/w) PVA-DMSO-water gels with different DMSO concentrations. (A), $0.104 \mathrm{mf} \mathrm{DMSO}$; (B) $0.277 \mathrm{mf}$ DMSO; (C), $0.582 \mathrm{mf}$ DMSO. Figures beside curves represent the temperature in ${ }^{\circ} \mathrm{C}$.

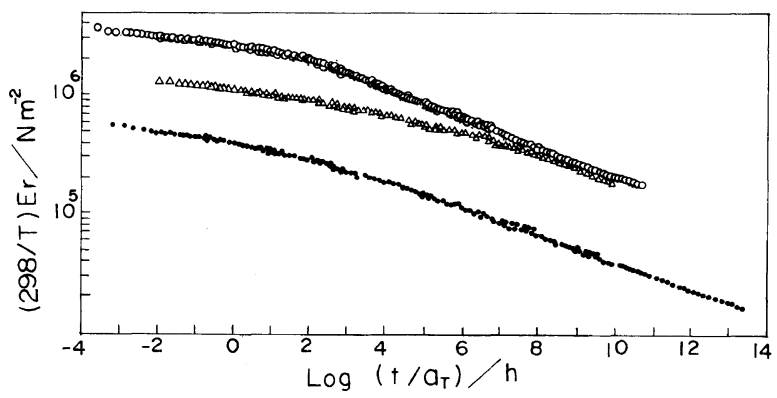

Figure 5. Master relaxation curves of $40 \%$ (w/w) PVA-DMSO-water gels with different DMSO concentrations. $0.104 \mathrm{mf}$ DMSO; O, $0.277 \mathrm{mf}$ DMSO; $\triangle, 0.582 \mathrm{mf}$ DMSO. 


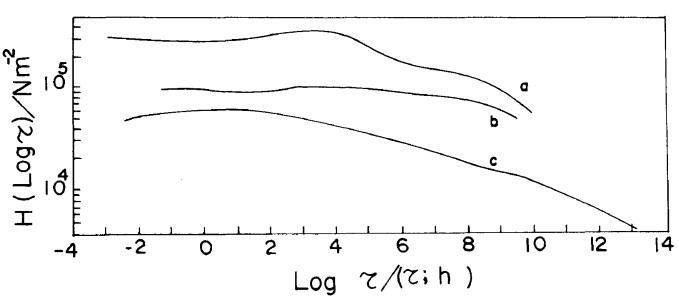

Figure 6. Relaxation spectra of $40 \%(w / w)$ PVADMSO-water gels with different DMSO concentrations. (a), 0.277 mf DMSO; (b), 0.582 mf DMSO; (c), $0.104 \mathrm{mf}$ DMSO.

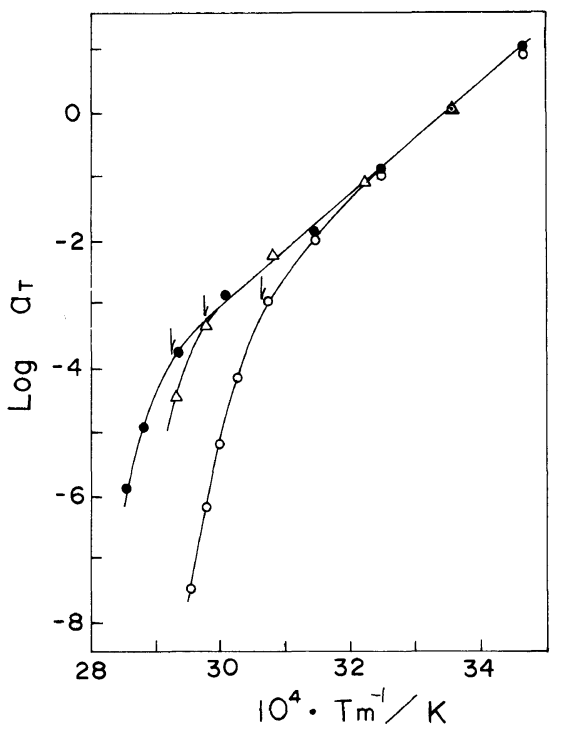

Figure 7. The relation between average shift factor $a_{\mathrm{T}}$ and $1 / T_{\mathrm{m}}$ for $40 \%(\mathrm{w} / \mathrm{w})$ PVA-DMSO-water gels with different DMSO concentrations. $\triangle, 0.104 \mathrm{mf}$ DMSO; - $0.277 \mathrm{mf}$ DMSO; $\bigcirc, 0.582 \mathrm{mf}$ DMSO. A downward arrow shows an inflexion point which corresponds to the internal melting of gels.

on a balance of crystallinity and the solubility. The decrease in $T_{\mathrm{m}}, \Delta H_{\mathrm{m}}$, beyond DMSO $0.277 \mathrm{mf}$ was attributed to the increase of free DMSO which shifts this balance to the solubility side. The increase in $T_{\mathrm{m}}$ and $\Delta H_{\mathrm{m}}$ with increasing DMSO concentration up to $0.277 \mathrm{mf}$ was attributed to the decrease of free water which did not form a hydrated compound with DMSO. When the free water content is higher, the gelling ability is lower.

Since interaction between water and DMSO is very strong, a mixture of water and DMSO shows peculiar characteristics. The structure of a hydrated compound of DMSO has been shown to be $\left(\mathrm{CH}_{3}\right)_{2} \mathrm{SO} \cdot 2 \mathrm{H}_{2} \mathrm{O}$ by viscometric ${ }^{14}$ and ultrasound velocity ${ }^{15}$ investigation. However, the neutron diffraction study has shown it to be $\left(\mathrm{CH}_{3}\right)_{2} \mathrm{SO} \cdot 3 \mathrm{H}_{2} \mathrm{O} .{ }^{16}$ Note that $0.277 \mathrm{mf}$ DMSO is the intermediate of these two compounds.

The fact that the relaxation spectrum of PVA gels containing DMSO showed a maximum at the DMSO concentration around $0.277 \mathrm{mf}$ (Figure 6) is understood as follows. The elastic modulus of thermo-reversible gels depends on the number and structure of crosslinking junction zones, and on the conformation of flexible chains which connect junction zones. A simple gel model, consisting of Langevin chains whose both ends are loosely fixed in crystalline junction regions in such a way that the segments near each end are bound densely in one of the junction region, has been proposed in order to explain the temperature dependence of elasticity of thermo-reversible gels. ${ }^{17}$ According to this model, the segments are assumed to be released from the junction zone with increasing temperature just as threads are released from a reel. When all the segments in one of the two ends are liberated from the junction zone, the chain is called a free chain in the theory of rubber elasticity, ${ }^{18}$ and would cease to contribute to the elasticity. Using this model, the elastic modulus of thermo-reversible gels can be calculated numerically by assuming the number $\mathscr{N}$ of molecular chains in a unit volume, the values of bonding energy $\varepsilon$, average end-to-end distance of Lengevin chains $r_{\mathrm{m}}$, an upper limit $v$ of the number of segments released from the junction zone before the chain ceases to contribute to the elasticity. The number of crosslinking junction zones will decrease with increasing DMSO content. The elastic modulus of thermo-reversible gels is proportional to the number of cross-linking junction zones if the conformations of flexible chains are the same. 
The presence of DMSO changes the ratio of entropic and energetic contributions to the elastic modulus. The entropic term increases with increasing length of flexible chains. ${ }^{19}$ At a certain DMSO concentration around $0.277 \mathrm{mf}$, three main factors, the number and structures of cross-linking junction zones, the conformation of flexible chains connecting junction zones, or $\varepsilon, r_{\mathrm{m}}, v, \mathcal{N}$ in the above-mentioned model which determine the elastic modulus, become optimum to realize the maximum of elastic modulus. Research should be conducted to determine the effects of DMSO on the conformation of PVA molecules in water, and on the structure of junction zones so that we may be able to understand further details.

\section{REFERENCES}

1. N. A. Peppas, Makromol. Chem., 176, 3433 (1975).

2. M. Watase and K. Nishinari, Polym. Comm., 24, 270 (1983).

3. M. Watase and K. Nishinari, Cryo-Letters, 4, 197 (1983).

4. M. Watase and K. Nishinari, Makromol. Chem., 186, 1081 (1985).

5. M. Watase and K. Nishinari, J. Polym. Sci., Polym. Phys. Ed., 23, 1803 (1985).
6. S. V. Rogozhin, V. I. Lozinsky, E. S. Vainerman, L. V. Domotenko, S. M. Mamtsis, S. A. Ivanova, M. I. Shtil'man, and V. V. Korshak, Dokl. Akad. Nauk., SSSR, 278, 129 (1984).

7. V. I. Lozinsky, L. V. Domotenko, E. S. Vainerman, A. M. Mamtsis, and S. V. Rogozhin, Polym. Bull., 15, 333 (1986).

8. F. Yokoyama, I. Masada, S. Shimamura, T. Ikawa, and K. Monobe, Colloid Polym. Sci., 264, 19 (1986).

9. T. Matsuo and H. Inagaki, Makromol. Chem., 53, 130 (1960)

10. D. Braun and E. Walter, Colloid Polym. Sci., 258, 376 (1980).

11. M. Watase and K. Nishinari, Rheol. Acta, 22, 580 (1983).

12. M. Watase and K. Nishinari, Polym. J., 20, 1125 (1988).

13. S. Okada, H. Hoshino, H. Urakawa, T. Kajiwara, and T. Ito, Polym. Prepr., Jpn., V. 37, No. 9, 3064 (1988).

14. S. A. Schichman and R. L. Amey, J. Phys. Chem., 75, 98 (1971).

15. D. E. Bowen, M. A. Priesand, and M. P. Eastman, J. Phys. Chem., 78, 2611 (1974).

16. D. H. Rasmussen and A. P. MacKenzie, Nature, 220, 1315 (1968).

17. K. Nishinari, S. Koide, and K. Ogino, J. Phys. (Paris), 46, 793 (1985).

18. L. R. G. Treloar, "The Physics of Rubber Elasticity," Clarendon Press, Oxford, 1975.

19. K. Nishinari, M. Watase and K. Ogino, Makromol. Chem., 185, 2663 (1984). 